\title{
Un codice arabo in caratteri ebraici dalla Trapani degli Abbate (Vat. ebr. 358)
}

\author{
Giuseppe Mandalà* \\ ILC - CCHS, CSIC - Madrid
}

\begin{abstract}
Un codice árabe en caracteres hebreos de la Trapani de los Abbate (Vat. ebr. 358). - El manuscrito Vat. ebr. 358 contiene el texto árabe escrito en caracteres hebreos de los apartados VI-X de la primera parte del Kitāb kāmil al-șinā'a al-țibbiyya ('El libro perfecto del arte medico'). Esta última es la enciclopedia médica compuesta antes de 367/977-978 por 'Alī ibn al-'Abbās alMajūsī (m. 384/994 [?]). En el presente artículo se apunta, por primera vez, al lugar de producción del manuscrito, Trapani (Ṭābaniš) en Sicilia. La ciudad aparece mencionada explícitamente en el colofón del manuscrito copiado el 26 de adar 5053 (13 de marzo 1293). Con este artículo se pretende contribuir al estudio de la circulación del conocimiento médico árabe entre los judíos sicilianos durante los siglos XIII a XV.
\end{abstract}

Palabras Clave: Árabe en escritura hebrea; medicina árabe; 'Alī ibn al-'Abbās al-Majūs̄̄; Kitāb kāmil al-șinā' a al-țibbiyya; Sicilia; ciencia; cultura manuscrita.

An Arabic Codex in Hebrew Characters from Abbate Trapani (Vat. ebr. 358). - The manuscript Vat. ebr. 358 contains the Arabic text written in Hebrew script of paragraphs VI-X of the first part of the medical encyclopedia Kitāb kämil al-șinā'a al-țibbiyya ('The perfect book of the medical art'). The work was composed before 367/977-978 by 'Alī ibn al-'Abbās al-Majūsī (d. 384/994 [?]). This article claims the place of production of the manuscript to be Trapani ( Trābaniš) in Sicily. The city is explicitly mentioned in the colophon that was copied on Adar 26, 5053 (March 13, 1293). This article expands our knowledge of the circulation of Arab medical science among Sicilian Jews (13-15 $\mathrm{c}$.).

Keywords: Arabic in Hebrew Script; Arabic Medicine; 'Alī ibn al-'Abbās al-Majūsī; Kitāb Kāmil al-Șinā'a al-Tibbiyya; Sicily; Science; Manuscript Culture.

\footnotetext{
"giuseppe.mandala@cchs.csic.es
} 
Negli ultimi decenni l'interesse nei confronti degli ebrei di Sicilia è cresciuto in maniera esponenziale, anche grazie alla messa in valore dell'ingente mole documentale conservata presso gli archivi siciliani ${ }^{1}$.

Questa emergenza, associata alle testimonianze delle fonti ebraiche, dovrà provocare un profondo dibattito sulle lingue utilizzate dagli ebrei siciliani, ma anche sul ruolo di mediatori culturali tra il mondo dell' Islam e l'Europa latina, specialmente durante i secoli XIII e XV ${ }^{2}$.

In questa occasione desidero attirare l'attenzione sulla circolazione del sapere medico arabo-islamico all'interno delle comunità ebraiche siciliane del basso Medioevo. Innanzitutto occorre notare che la presenza di opere mediche non risulta particolarmente numerosa negli elenchi di manoscritti ebraici copiati in Sicilia $^{3}$. Inoltre, a dispetto della forte e continua presenza di medici ebrei nella documentazione archivistica, la medicina è poco presente nei ventotto inventari latini di libri appartenuti ad ebrei siciliani ${ }^{4}$.

Tuttavia occorre rilevare sin dalle prime battute che questo apparente silenzio cela una realtà ben più complessa ed articolata che si presta a futuri approfondimenti e scoperte. Per quanto concerne la nostra indagine, per il tramite di esponenti delle comunità ebraiche di Sicilia abbiamo traccia della circolazione delle tre maggiori enciclopedie mediche arabe medievali note in Occidente ${ }^{5}$.

${ }^{1}$ Sh. Simonsohn, The Jews in Sicily, vol. I-XVIII (Leiden-New York-Köln 1997-2010).

${ }^{2}$ Per uno status quaestionis rimando a B. GRÉvin, G. MANDALÀ, «Le rôle des communautés juives siciliennes dans la transmission des savoirs arabes en Italie, $\mathrm{XIII}^{\mathrm{e}}-\mathrm{XV}^{\mathrm{e}}$ siècle», in La frontière méditerranéenne (15 $-17^{e}$ siècles). Échanges, circulations et affrontements, Tours, 17-19 June 2009, Centre d'Études Supérieures de la Renaissance, eds. A. Fuess, B. Heyberger, Ph. Vendrix (Turnhout, in corso di stampa).

${ }^{3}$ C. Roth, «Jewish Intellectual Life in Medieval Sicily », JQR 47-48 (1956-58), pp. 317-335: 330-331 e n. 32; G. TAMANI, «Manoscritti ebraici copiati in Sicilia nei secoli XIV-XV», Henoch 15 (1993), pp. 107-113; M. BeIT-ARIÉ, Additamenta to G. Tamani's Article on Hebrew Manuscripts Copied in Sicily, Henoch 15 (1993), pp. 359-361; N. ZeLDES, «Diffusion of Sicilian Exiles and Their Culture as Reflected in Hebrew Colophons», Hispania Judaica Bulletin 5 (2007), pp. 303332; Simonsohn, The Jews in Sicily, XVIII, pp. 12121-12126.

${ }^{4} \mathrm{Si}$ veda ad esempio l'inventario dei beni di Vita Xifuni, medico e cittadino di Palermo, che comprendeva «1. Item librum unum medicine in carta bonbicis; 2. Item XXII pecia librorum medicine et aliarum scienciarum ad unum folium in carta bonbicina; 3. Item unum librum muriscum; 4. Item XVIII libros ad unum quartum foliu» (Palermo, 1 dicembre 1443), cfr. H. BRESC, Livre et société en Sicile (1299-1499) (Palermo 1971), pp. 63-69: 64, 170.

${ }^{5}$ Come rilevato da Françoise Micheau, al-Majūsī può essere considerato uno degli enciclopedisti arabi «dont la portée va se faire sentir en Orient comme en Occident», Fr. MicheAu, 'Alī ibn al-'Abbās al-Mağūsī et son milieu, in Constantine the African and 'Alī ibn al-'Abbās alMağūsì. The Pantegni and Related Texts, eds. Ch. Burnett, D. Jacquart (Leiden-New York-Köln 
E mi riferisco in primo luogo al Kitāb al-ḥāwī fì l-țibb (Liber continens) di Abū Bakr Muḥammad b. Zakariyyā' al-Rāzī (250-313/854-925 o 323/935), tradotto in latino da Faraj ben Sālim da Agrigento sulla copia offerta dal sultano hafș̣ide al-Mustanșir (647-675/1249-1277) a Carlo I d'Angiò (1226-1285). La traduzione commissionata dal sovrano angioino ha inizio intorno al 2 settembre 1278 e termina il 31 agosto 1282, coinvolgendo un nutrito apparato di copisti, miniatori, gregari e aiutanti. Due testimoni angioini della traduzione, contemporanei e prodotti a corte, sono attualmente conservati a Paris, Bibliothèque nationale de France, Lat. 6912, 1-5; Città del Vaticano, Biblioteca Apostolica Vaticana, Lat. 2398-2399 .

In Sicilia, come in ogni dove, circola anche il Canone (al-Qānūn fí l-țibb) di Avicenna/Ibn Sīnà (370-428/980-1037), un testo che, suddiviso in cinque libri, è tradotto in ebraico (libri II-V) da Natan ha-Me'ati (a. 1279), traduzione successivamente stampata a Napoli in tre volumi (a. 1491-92). Parti dell'opera sono state tradotte anche da Zeraḥya b. Yișhaq Gracian/Hen (a. 1249), e da Yosef b. Yehošu a ha-Lorqi (a. 1408, libro I incluso nell'edizione a stampa di Napoli alla fine del XV secolo) ${ }^{7}$. Del Canone in ebraico conosciamo 111 testimoni completi o parziali, e come sottolineato da Nancy Siraisi: «the most extensive use of the Canon outside the universities in Western Europe during the Middle Ages was unquestionably by Jewish physicians» ${ }^{8}$. Quanto alla sua circolazione in Sicilia, dai documenti d'archivio apprendiamo che il Canone, nella sua interezza o in

1994), pp. 1-15: 1; sulle enciclopedie mediche arabe note nell'Occidente medievale rimando a D. JACQUART, Fr. MicheAu, La médecine arabe et l'Occident médiéval (Paris 1990).

${ }^{6}$ M. STEINSCHNEIDER, Die hebräischen Übersetzungen des Mittelalters und die Juden als Dolmetscher (Berlin 1893), pp. 721, 974-975; A. DANEu LATTANZI, «Una «bella copia» di al-Ḥāwī tradotto dall'arabo da Farag Moyse per Carlo I d'Angiò (Ms. Vat. Lat. 2398-2399). Contributo alla storia della miniatura dello scorcio del sec. XIII a Napoli», in Miscellanea di studi in memoria di Anna Saitta Revignas (Firenze 1978), pp. 149-169; P. SUPINo MARTINI, «Linee metodologiche per lo studio dei manoscritti in litterae textuales prodotti in Italia nei secoli XIII-XV», Scrittura e civiltà 17 (1993), pp. 43-101: 45-70; K.-D. FisCHER, U. WEISSER, «Das Vorwort zur lateinischen Übersetzung von Rhazes' Liber continens (1282). Text, Übersetzung und Erläuterungen», Medizinhistorisches Journal 21 (1986), pp. 211-241.

${ }^{7}$ STEINSChNeIDER, Die hebräischen Übersetzungen des Mittelalters, pp. 678-679 (sulla traduzione di Natan ha-Me'ati); pp. 111-124 e 681 (sulla traduzione di Zerahyya Hen); pp. 681682 (sulla traduzione di Yehošu'a ha-Lorqi); B. Richler, «Manuscript of Avicenna's Kanon in Hebrew Translation: A Revised and Up-to-date List», Koroth 8 (1982), pp. 145-168 (in ebraico); J. Leibowitz, «The preface by Nathan ha-Meati to his Hebrew translation of Avicenna's Canon (1279)», Koroth 14 (2000), pp. 149-154;

${ }^{8}$ N. G. SIRAISI, Avicenna in Renaissance Italy: The Canon and Medical Teaching in Italian Universities after 1500 (Princeton 1987), p. 48. 
alcune sue parti, è copiato a Palermo ${ }^{9}$ e venduto a Trapani (a. 1444), Polizzi (a. 1481) e Palermo (a. 1492) ${ }^{10}$.

All'appello, fino ad oggi, mancava il Kitāb kāmil al-șinā'a al-țibbiyya ('Il libro perfetto dell'arte medica'), enciclopedia medica di 'Alī ibn al-'Abbās alMajūsī (m. 384/994 [?]; l'opera è composta ante 367/977-78), nota anche come al-Kitāb al-malakī perché composto in onore del principe (in arabo malik) buyide 'Aḍud al-Dawla (367-372/977-982). Questa lacuna può essere ora colmata grazie al Vaticano ebraico 358.

\section{IL MANOSCRITTO}

Il Vaticano ebraico 358 consta di 202 ff.; il manoscritto è in pergamena e carta senza filigrana; i fascicoli sono composti da nove bifogli; il bifoglio esterno e quello interno di ogni fascicolo è in pergamena. Misura 256 x 173 mm (177 x $113 \mathrm{~mm}$ del campo scrittorio), mentre $\mathrm{i}$ fogli di pergamena sono più corti di 2-3 mm ${ }^{11}$. La grafia è una semicorsiva sefardita in inchiostro marrone; le lettere omografe, a volte, sono distinte con un punto soprascritto (es.: k/h; d/d ; ș/d), mentre la $t \bar{a}^{\prime}$ marbūta è segnata da una $h e^{\prime}$ con due punti soprascritti; in alcuni casi le consonanti ebraiche recano vocali arabe (es. rahima-hu con damma). Come consueto i numerali hanno dei punti soprascritti. Ricorrente è il nesso aleph-lamed per indicare l'articolo arabo al-.

Il manoscritto include i capitoli VI-X della prima parte del Kitāb kāmil alșinā' a al-țibbiyya di 'Alī ibn al-'Abbās al-Majūs̄̄, un'opera suddivisa in una parte teorica e una pratica, entrambe in dieci libri ${ }^{12}$. Come noto il testo è stato

\footnotetext{
${ }^{9}$ Una copia incompleta del Canone, copiata a Palermo da Šemu'el ben Yosef ha-rofe' (XIVXV sec.), è conservata a Modena, Biblioteca Estense, a. N. 5. 15. F 14947, cfr. Simonsohn, The Jews in Sicily, XVIII, p. 12124.

${ }^{10}$ Sulla circolazione del testo nella Sicilia ebraica del XV sec. cfr. H. BRESC, Arabi per lingua ebrei per religione. L'evoluzione dell'ebraismo siciliano in ambiente latino dal XII al XV secolo (Messina 2001), pp. 58-59; M. Bevilacqua Krasner, «Sicilia e Meridione. Rapporti e scambi tra le comunità durante il XIV e XV secolo», Sefer Yuḥasin 21-22 (2005-2006), pp. 25-38: 35-37; GrÉvin, Mandalà, «Le rôle des communautés juives siciliennes».

${ }^{11}$ Per la descrizione codicologica seguo Hebrew Manuscripts in the Vatican Library. Catalogue. Compiled by the Staff of the Institute of Microfilmed Hebrew Manuscripts, Jewish National and University Library, Jerusalem, Edited by B. RicHLER, Palaeographical and Codicological Descriptions M. Beit-ArIÉ in collaboration with N. PAsternaK (Città del Vaticano 2008), pp. 302-303.

${ }^{12}$ Per il testo arabo dei capitoli VI-X del prima parte si faccia riferimento all'edizione: 'Alī b. al-'Abbās al-Majūsī, Kāmil al-șinā'a al-țibbiyya, I-II (Il Cairo/Būlāq 1294/1877; rist. Frankfurt am Mein 1996), I, pp. 217-434; si veda anche il facsimile del ms. Istanbul, University Library, A.
} 
«tradotto» in latino sotto il nome di Pantegni (la prima parte è chiamata Theorica, la seconda Practica) da Costantino Africano (m. prima del 1098/9), un monaco di Monte Cassino originario di Cartagine, e successivamente da Stefano di Antiochia con il titolo di Liber regalis o Regalis dispositio (intorno al 1127) ${ }^{13}$.

Del testo arabo del Kitāb kāmil al-șinā'a al-țibbiyya esistono anche una versione in ebraico e quattro traslitterazioni parziali in lettere ebraiche di cui il manoscritto Vaticano ebr. 358 è il testimone più antico ${ }^{14}$. La pratica di traslitterare un testo arabo in caratteri ebraici è abbastanza diffusa negli ambienti ebraici arabofoni del Medioevo, e trova la sua giustificazione prima nella immediata fruizione linguistica di un testo letto attraverso i segni identitari più tangibili: le lettere ebraiche ${ }^{15}$. Senza dubbio il Vat. ebr. 358 fa parte di quella folta schiera di opere scientifiche, ma non solo, arabe in caratteri ebraici circolanti negli ambienti ebraici del Medioevo ${ }^{16}$; nella definizione di questo tipo di testi vige una certa ambiguità, che oscilla tra parametri di classificazione di tipo socio-culturale fondati sulla pertinenza e/o appartenenza alla cultura ebraica delle opere in questione, a fianco a definizioni che fanno appello a modelli più apparentemente linguistici ${ }^{17}$.

Y. 6375, riprodotto anastaticamente in 'Alī ibn al- 'Abbās al-Majūsī, Kāmil al-șinā'a al-țibbiyya. The Complete Medical Art, I-III (Frankfurt am Main 1985), I, pp. 251-513.

${ }^{13}$ Ch. Burnett, D. JacQuart, «Preface», in Constantine the African and 'Alī ibn al-'Abbās al-Mă̌ūsī, pp. VII-IX: VII-VIII.

${ }^{14}$ R. BARKAI, «The Judaeo-Arabic and Hebrew Versions of the Kitāb kāmil aș-șinā' $a »$, in Constantine the African and 'Al̄̄ ibn al- 'Abbās al-Mağūsī, pp. 57-70: 58-62 (per la tradizione araba in caratteri ebraici), 63-70 (per la versione in ebraico). Il Vat. ebr. 358 è già segnalato in M. STEINSCHNEIDER, «Schriften der Araber in hebräischen Handschriften: ein Beitrag zur arabischen Bibliographie», Zeitschrift der Deutschen Morgenländischen Gesellschaft 47 (1893), pp. 335-384: 341.

${ }^{15}$ Sul valore identitario dell'alfabeto ebraico cfr. B. H. HARY, Multiglossia in Judeo-Arabic. With and Edition, Translation, and Grammatical Study of the Cairene Purim Scroll (Leiden-New York-Köln 1992), pp. 72-73.

${ }^{16}$ STEINSCHNEIDER, «Schriften der Araber in hebräischen Handschriften»; ID., «An Introduction to the Arabic Literature of the Jews», JQR 12 (1900), pp. 481-501: 499-501; Y. Tzvi LangermanN, «Transcriptions of Arabic Treatises into the Hebrew Alphabet: an Underappreciated Mode of Transmission», in Tradition, Transmission, Transformation. Proceedings of Two Conferences on Pre-modern Science held at the University of Oklahoma, eds. F. J. RAGEP, S. P. RAGEP (Leiden 1996), pp. 247-260; ID. Y. Tzvi LangermanN, «Arabic Writings in Hebrew Manuscripts: A Preliminary Relisting», Arabic Sciences and Philosophy 6 (1996), pp. 137-160.

${ }^{17}$ SteinschneideR, «Schriften der Araber in hebräischen Handschriften», pp. 335-336; R. KunNe, «Una versión aljamiada del "Secreto de Hipócrates"», Sefarad 46 (1986), pp. 253-269; P. Fenton, «Judaeo-Arabic Literature», in Religion, Learning and Science in the Abbasid Period, eds. M. J. L. Young, J. D. Latham, R. B. Serjeant (Cambridge 1990), pp. 461-476: 462-463; 471-472; Tzvi Langermann, «Arabic Writings in Hebrew Manuscripts, pp. 137-141; J. Blau, The 
Dal punto di vista linguistico occorre precisare che il nostro codice reca un testo in arabo classico traslitterato in una grafia ebraica che lascia emergere tratti tipici del giudeo-arabo (qui inteso come medio-arabo in caratteri ebraici), ossia secondo la definizione proposta da J. Blau: «Classical Arabic with Middle/Neo Arabic admixture» ${ }^{18}$; dal punto di vista socio-culturale si tratta di un'opera medica in arabo, composta da un autore arabo-islamico del X sec., copiata e fruita in lettere ebraiche negli ambienti ebraici siciliani del basso Medioevo.

Come già segnalato da Angelo Michele Piemontese, il Vaticano ebraico 358 fa parte del più antico nucleo di codici «giudeo-arabi» di Sicilia confluito nelle raccolte vaticane (e ivi presente sin dal XVI secolo), ossia un gruppo di manoscritti esplicitamente copiati nell'isola e/o precedentemente appartenuti ad ebrei/conversi siciliani, quali Guglielmo Raimondo Moncada alias Flavio Mitridate o Antonio Flammino Biaxander, solo per citare i più noti ${ }^{19}$.

Il manoscritto ebraico 358 è stato già esaminato sia dai catalogatori della Biblioteca Apostolica Vaticana ${ }^{20}$, sia da chi si è occupato della tradizione manoscritta «giudeo-araba» dell'opera ${ }^{21}$, tuttavia il luogo di composizione è rimasto fino ad oggi totalmente incompreso ${ }^{22}$. Il manoscritto reca i seguenti incipit e colophon:

Incipit (f. 1r, ll. 1-6; fig. 1)

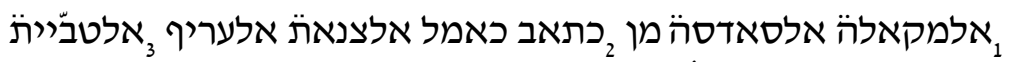

$$
\begin{aligned}
& \text { והי אלאמראץ }{ }_{4}{ }_{4} \\
& \text { וֹלאثון באבא. אלבאב אלא פי פי גמלה אלה אלכלאם } \\
& \text { ען אלאמר אלטביעי. }
\end{aligned}
$$

Emergence and Linguistic Background of Judaeo-Arabic. A Study of the Origins of Neo-Arabic and Middle Arabic. Third Revised Edition (Jerusalem 1999), pp. 25-26, 46-47.

${ }^{18}$ BLAU, The Emergence and Linguistic Background of Judaeo-Arabic, pp. 25, 239. O anche: «The four manuscripts known to us are quite close to the Arabic original, although they differ among themselves in their levels of linguistic sophistication and their relation to Classical Arabic», BARKAI, «The Judaeo-Arabic and Hebrew Versions», pp. 59-60, 62.

${ }^{19}$ A. M. Piemontese, «Codici giudeo-arabi di Sicilia», in Ebrei e Sicilia, eds. N. Bucaria, M. Luzzati, A. Tarantino (Palermo 2002), pp. 179-183: 180.

${ }^{20}$ Hebrew Manuscripts in the Vatican Library, pp. 302-303.

${ }^{21}$ BARKAI, «The Judaeo-Arabic and Hebrew Versions», pp. 58-60.

${ }^{22}$ Secondo Barkai: «Ṭarābanš ar-Rātba on the seacoast... in the eastern Mediterranean basin» cfr. BARKAI, «The Judaeo-Arabic and Hebrew Versions», pp. 59, 62; Secondo i catalogatori della Vaticana: טראבנש (Tunisia?)», cfr. Hebrew Manuscripts in the Vatican Library, p. 302. 


\section{Trascrizione}

${ }_{1}$ al-Maqāla al-sādisa min ${ }_{2}$ Kitāb kāmil al-șinā'a al- 'arīf ${ }^{23}{ }_{3} a l$-țibbiyya wa-hiya al-amrāọ ${ }_{4}$ wa-asbābu-hā wa-l-a 'rạ̣̄ al-tābi' a ${ }^{24}$ la-ha, wa-hiya 1. w. ${ }_{5}^{25}$ sitta wa-talātūun bāban. al-Bāb al-a(wwal) fī jumlat al-kalām ${ }_{6}$ 'an alumūr al-hārija 'an al-amr al-țabīi $\overline{1}$.

\section{Traduzione}

${ }_{1}$ Il sesto capitolo del ${ }_{2}$ Libro perfetto dell'arte [per] la conoscenza ${ }_{3}$ medica ed esso comprende le malattie ${ }_{4}$ e le loro cause, e i relativi accidenti naturali, [suddivisi] in $36{ }_{5}$ trentasei paragrafi. Il primo paragrafo [contiene] l'insieme della trattazione ${ }_{6}$ riguardo ciò che sopravviene per causa naturale.

Colophon (f. 202r, ll. 20-24; fig. 2)

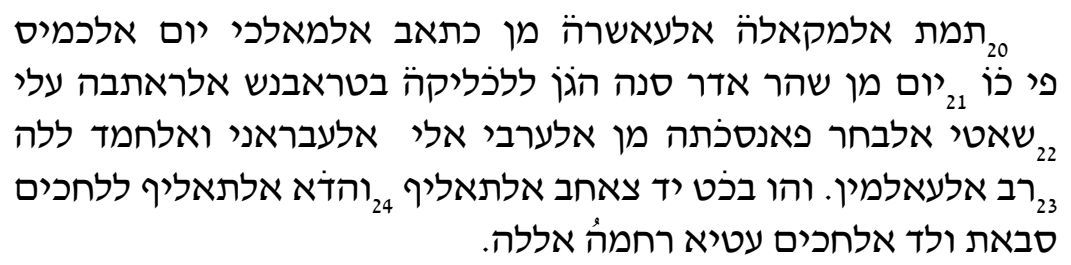

Trascrizione

${ }_{20}$ Tammat al-maqāla al- 'āšira min Kitāb al-mālak̄̄ yawm al-ḩamīs fī sitta wa-'išrīn ${ }_{21}$ yawm min šahr adar sanat hams ālāf wa-țalāt wa-hamsīn li-lhalīqa bi-Ṭrābaniš al-rātiba 'alà ${ }_{22}$ šâțī al-baḥr fa-ansahtu-hu min al- 'arabī ilà 1-'ibrānī wa-l-ḥamd li-Llah ${ }_{23}$ rabb al-'ālamīn wa-huwa bi-ḩațt yad șāḥib alta' lịf ${ }_{24}$ wa-haḍa al-ta' līf li-l-ḥakīm Sabbāt wuld al-ḥakīm 'Ațiyyā raḥimahu Allah.

${ }^{23} \mathrm{Sic}$, invece di un più probabile $[f i]$ 'urf, «connaissance», sul valore del termine cfr. A. de Biberstein Kazimirski, Dictionnaire arabe-français, I-II (Paris 1860), II, p. 226. È tuttavia possibile che lo scriba abbia frainteso l'espressione al-ma 'rüf bi-l-Malaki «noto come Il [libro] regale», che ricorre nell'incipit del testo in arabo e per cui si veda infra.

${ }^{24}$ Sic, invece di wa-l-a'rāọ al-țabì'iyya. Sul valore di 'araḍ, plur. a 'rāọ, «accident (phil.)», si veda F. Corriente, A Dictionary of Andalusi Arabic (Leiden 1997), pp. 349-350.

${ }^{25} \mathrm{Sic}$, il numerale 36 è indicato due volte, la prima volta dalla sequenza di lettere $l . w$., la seconda per esteso. 


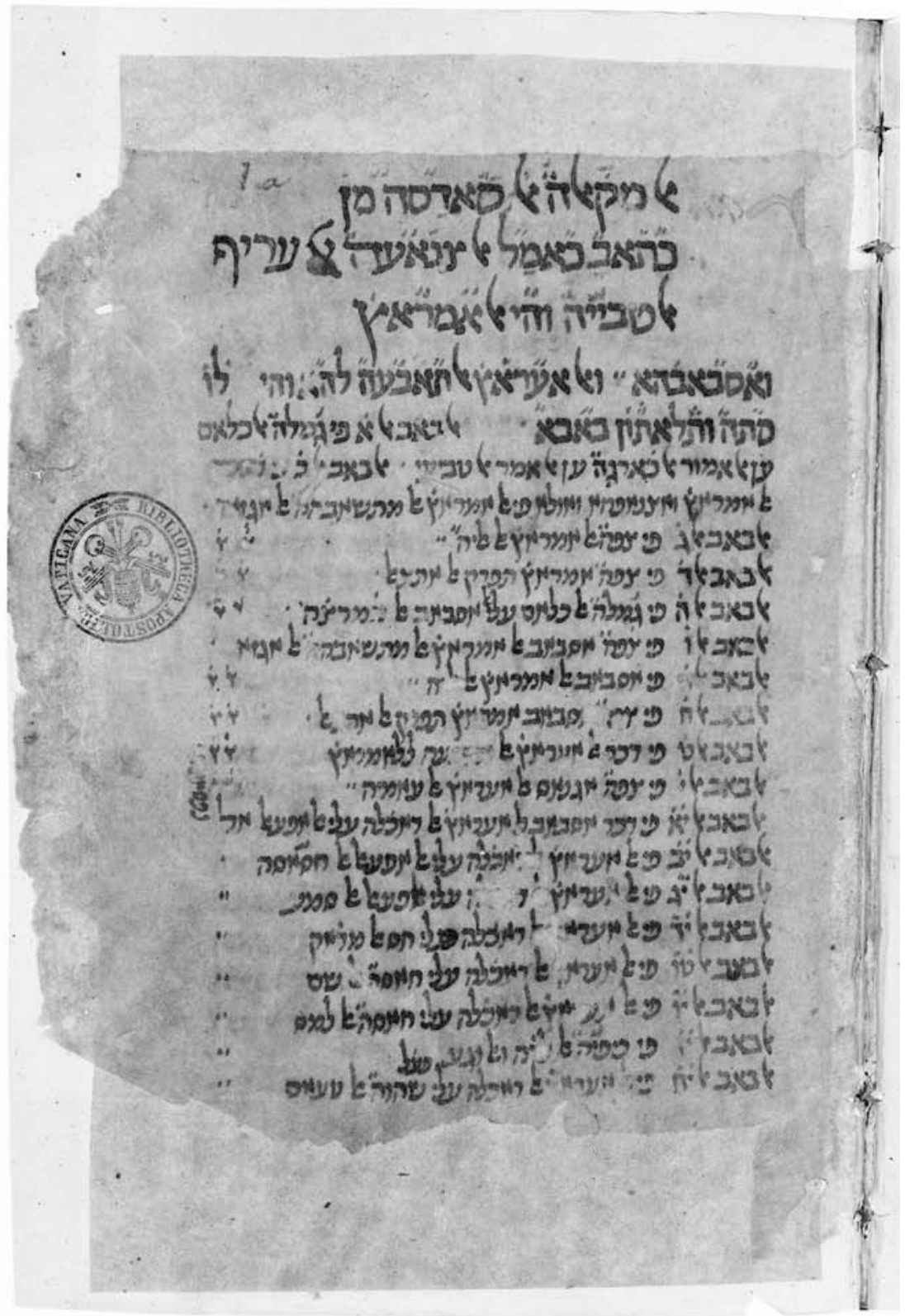

Vat. ebr. 358, f. 1r (@ 2011 Biblioteca Apostolica Vaticana). 


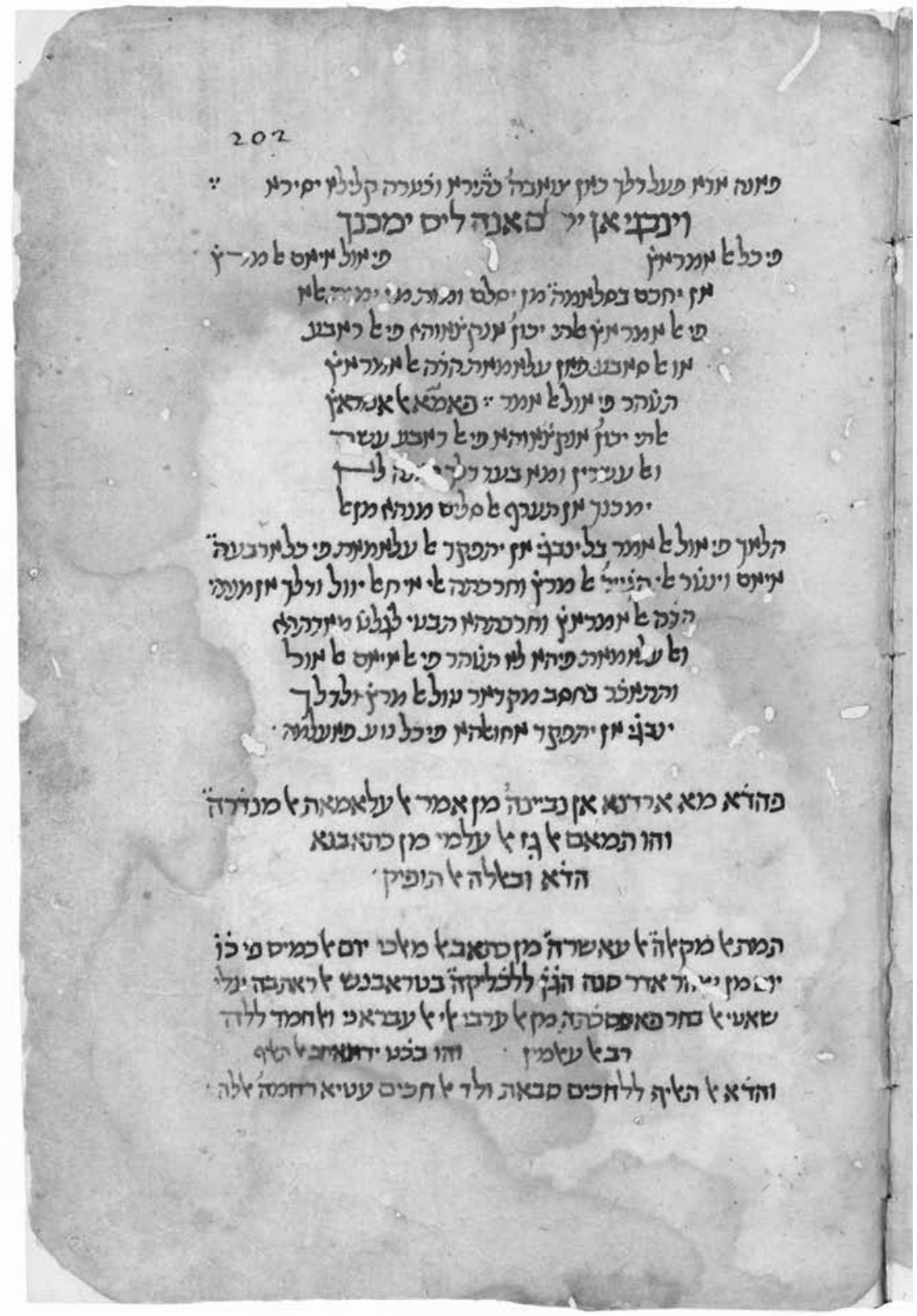

Vat. ebr. 358, f. 202r (@ 2011 Biblioteca Apostolica Vaticana). 


\section{Traduzione}

${ }_{20} \mathrm{E}$ terminato il decimo capitolo de Il libro regale [Kitāb al-mālakī] giovedì $26_{21}$ del mese di adar dell'anno 5053 della creazione [13 marzo 1293] in Trapani situata sulla ${ }_{22}$ riva del mare, e lo ho traslato dall'arabo all'ebraico, grazie a Dio ${ }_{23}$ signore degli universi, ed esso [scil. il codice] è di mano del proprietario della copia, ${ }_{24}$ e questa copia appartiene al medico Sabbāt figlio del medico 'Ațiyyā, che Dio abbia misericordia di lui.

L'incipit del nostro manoscritto si scosta, in alcuni punti, sia dalla tradizione manoscritta che dall'edizione a stampa; per scrupolo filologico sarà di qualche utilità riportarne il testo:

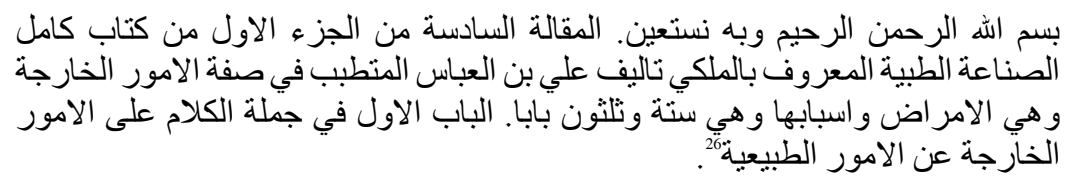

Bi-smi Llāh al-raḥm(ā)n al-raḥīm wa-bi-hi nasta'īnu. al-Maqāla alsādisa min al-juz' al-awwal min Kitāb kāmil al-șinā' 'a al-țibbiyya al-ma' rūf bi-l-Malakī ta' līf 'Alī b. al- 'Abbās al-mutațabbib fī șifat al-umūr al-hārija wa-hiya al-amrạ̣̄ wa-asbābu-ha wa-hiya sitta wa-țal(ā)țūn bāban. al-Bāb al-awwal fì jumlat al-kalām 'alà l-umūr al-ḩārija 'an al-umūr al-țabī'iyya.

Nel nome di Dio, clemente e misericordioso, a Lui facciamo ricorso. Il sesto capitolo della prima parte de Il libro perfetto dell'arte medica, noto come Il [libro] regale, opera di 'Alī b. al-'Abbās l'esperto di medicina $(a l-m u t a t ̦ a b b i b)^{27}$, riguardo ciò che sopravviene, ossia le malattie e le loro cause, in trentasei paragrafi. Il primo paragrafo [contiene] l'insieme della trattazione riguardo ciò che sopraggiunge per cause naturali.

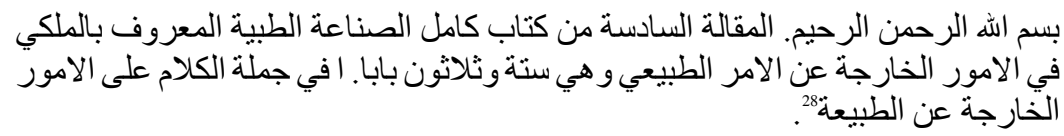

${ }^{26}$ Istanbul, University Library, A. Y. 6375, 'Alī b. al- 'Abbās al-Majūsī, Kāmil al-ṣinā'a alțibbiyya, I, p. 251 (facsimile).

${ }^{27}$ Sul valore del termine si vedano le osservazioni M. MeYerhof, «Esquisse d'histoire de la pharmacologie et botanique chez les musulmans d'Espagne», Al-Andalus 3 (1935), pp. 1-41: 24.

28 'Alī b. al-'Abbās al-Majūsī, Kāmil al-șinā'a al-țibbiyya, I, p. 217 (edizione Būlāq). 
Bi-smi Llāh al-raḥm(ā)n al-raḥ̄m. al-Maqāla al-sādisa min Kitāb kāmil al-șinā'a al-țibbiyya al-ma'rūf bi-l-Malakī fī l-umūr al-hārija 'an al-amr alțabī'ī wa-hiya sitta wa-țalātūna bāban. a(l-Awwal) fī jumlat al-kalām 'alà al-umūr al-ḩārija 'an al-țabī'a.

Nel nome di Dio, clemente e misericordioso. Il sesto capitolo de Il libro perfetto dell'arte medica, noto come Il [libro] regale, riguardo ciò che sopravviene per causa naturale, in trentasei paragrafi. Il [primo paragrafo contiene] l'insieme della trattazione riguardo ciò che sopraggiunge dalla natura.

Il colophon ci informa con tutta evidenza che il codice è stato copiato a Trapani, città menzionata col suo nome di forma araba Trābaniš (a volte con alif prostetica Itrāabaniš, e così ad esempio documentata nelle lettere della Ghenizà ${ }^{29}$; il toponimo è introdotto dalla preposizione $b i$-/be-, chiara interferenza medio-araba. In margine occorre notare che ritroviamo la locuzione «situata sulla riva del mare» (al-rātiba 'alà šâți al-baḥr $)^{30}$, attribuita a Palermo, nel colophon giudeo-arabo di un altro manoscritto siciliano: il Vaticano ebr. 361 (f. $166 \mathrm{v})^{31}$. Da notare che il titolo dell'opera reca al-mālakī con alif di prolungamento, e non al-malakī come più comunemente vulgato. La data giovedì 26 del mese di adar dell'anno 5053 della creazione corrisponde al 13 marzo 1293 anno

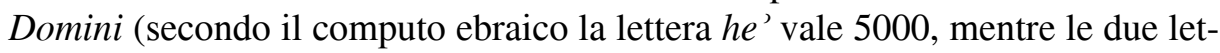
tere gimel e nun con il punto soprascritto indicano 3 e 50 rispettivamente) ${ }^{32}$.

\footnotetext{
${ }^{29}$ Per uno spoglio delle attestazioni arabo-sicule rimando a G. CARACausi, Dizionario onomastico della Sicilia (Palermo 1993-94), II, p. 1642 s. v. Trapani; M. GIL, «Sicily 827-1072, in light of the Geniza documents and parallel sources», in Gli ebrei in Sicilia sino all'espulsione del 1492. Italia Judaica V, Atti del V convegno internazionale (Palermo, 15-19 giugno 1992) (Roma 1995), pp. 96-171: 148; M. Ben SASSON, The Jews of Sicily 825-1068. Documents and Sources (Jerusalem 1991), p. 431 (ivi da emendare Itrāabališ/Tripoli in Ițräbaniš/Trapani); N. Zeldes, M. Frankel, «Trade with Sicily. Jewish Merchants in Mediterranean Trade in the $12^{\text {th }}$ and $13^{\text {th }}$ Centuries» (in ebraico), Michael 14 (1997), pp. 89-137: doc. n. 4 (a. 1208); n. 5 (1194-1260). In Beniamino da Tudela (seconda metà del XII sec.) il nome ebraico della città è già טראפנה (Träpana), cfr. The Itinerary of Benjamin of Tudela, Critical Text, Translation and Commentary by M. N. AdLER (London 1907), p. 71 (testo ebraico). Il suffisso -iš della forma araba è interpretato come un residuo di un locativo plurale latino cfr. G. B. PELLEGRINI, Ricerche sugli arabismi italiani con particolare riguardo alla Sicilia (Palermo 1989), p. 148.

${ }^{30}$ L'espressione sembrerebbe riecheggiare היושבת על מבואות ים in Ezechiele 27, 3: «Dì a Tiro, alla città situata all'approdo del mare». Si noti anche che la parola araba šățī, 'riva, sponda', non nota la hamza con una alif finale.

${ }^{31}$ Per il Vat. ebr. 361 cfr. D. V. Proverbio, «Manoscritti scientifici giudeo-arabi (praeter lexica) nella serie dei codici Vaticani ebraici. Inventario analitico», in Miscellanea Bibliothecae Apostolicae Vaticanae 8 (Città del Vaticano 2001), pp. 351-405: 358.
}

${ }^{32}$ E. MAHLER, Handbuch der jüdischen Chronologie (Leipzig 1916), p. 568. 
Quanto al lessico, il copista utilizza una terminologia tecnica arabo-islamica: la copia è definita $t a^{\prime} l i \bar{f} f^{33}$, e il suo proprietario șăhi $i b^{34}$, mentre il verbo utilizzato per indicare la traslitterazione dall'arabo all'ebraico è nasaha alla IV forma ${ }^{35}$; non ultimo i maschili $b i-l$ - 'arabī e $b i-l$ - 'ibrān̄ farebbero pensare ad un uso linguistico medio-arabo. Occorre rilevare anche che le formule religiose rimandano ad un lessico puramente islamico (Allāh rabb al- 'alamīn; rahima-hu $\left.A l l \bar{a} h^{36}\right)$, indice di una certa «islamizzazione» dello scriba.

Sabbāt figlio di 'Ațiyyā è al contempo copista e proprietario del manoscritto, sicuro indice di una certa cultura e agiatezza economica; poco o nulla è possibile dire su di lui, eccezion fatta che per qualche osservazione sul nome, la genealogia e il mestiere. Entrambi i nomi ricorrono nell'onomastica ebraico-sicula, nelle forme latine $S a b(b) e t$, Sab(b)eti (> ebr. Šabbat, Šabbetay) e il diminuitivo Sab(b)atinus, e Atia, che è nome arabo in uso presso gli ebrei arabofoni ${ }^{37}$. Sabbāt si dichiara wuld, un termine che significa 'nato, procreato', ossia 'figlio' o più genericamente 'discendente' (a volte 'illegittimo' ${ }^{38}$; il termine wuld, che corrisponde al più classico walad, è utilizzato nei nasab ('genealogie') maghrebini come sinonimo di ibn/ben ('figlio')"

33 «Composition, compilation, work (of prose or poetry)» cfr. A. GACEK, The Arabic Manuscript Tradition: A Glossary of Technical Terms and Bibliography (Leiden-Boston-Köln 2001), p. 8.

${ }^{34}$ Il termine era stato letto da Barkai: «șahāāb» cfr. BARKAI, «The Judaeo-Arabic and Hebrew Versions», p. 59 n. 9; dai catalogatori della Vaticana: «חב] [.], cfr. Hebrew Manuscripts in the Vatican Library, p. 303.

${ }^{35}$ Alla IV forma col significato di «annuler», ma anche «transcrir, copier», o meglio «trasladar escritura» seguendo la definizione data da Pedro de Alcalá, cfr. R. Dozy, Supplément aux dictionnaires arabes, I-II (Leiden-Paris $1927^{2}$ ), II, p. 666; anche J. BLAU, Dictionary of Mediaeval Judaeo-Arabic Texts (Jerusalem 2006), p. 692. Il verbo era stato letto da BARKaI: «ashatata [sic]», cfr. BARKAI, «The Judaeo-Arabic and Hebrew Versions», p. 59 n. 9; dai catalogatori della Vaticana: «פאכסכ"תה, cfr. Hebrew Manuscripts in the Vatican Library, p. 303.

${ }^{36} \mathrm{Si}$ noti che in entrambi i casi la parola Allāh è scritta senza alif di prolungamento, forse indice di una diversa accentuazione?

${ }^{37}$ Come noto Šabbat è nome ebraico che si attribuisce a chi è nato di «sabato». Per attestazioni siciliane si veda ad esempio Simonsohn, The Jews in Sicily, II, doc. 383, p. 663; doc. 966, p. 1044; doc. 857, pp. 969-970; doc. 398, p. 672.

${ }^{38}$ Biberstein Kazimirski, Dictionnaire arabe-français, II, p. 1602.

39 «Ad ogni modo questo uso sporadico di wuld non ha più luogo, quando dopo il padre continua l'enumerazione degli antenati nel nasab», cfr. L. CAETANI, G. GABRIELI, Onomasticon arabicum ossia Repertorio alfabetico dei nomi di persona e di luogo contenuti nelle principali opere storiche, biografiche e geografiche stampate..., I-II (Roma 1915), I, p. 135. 
Sia lui che il padre sono due hakìm, una parola araba che indica il 'filosofo' e per estensione anche il 'medico' ${ }^{40}$ : un achim medic(us) compare al capezzale di Guglielmo II, intento a scrutarne le urine, in una «glossa» latina alla miniatura del pianto di Palermo per la morte del re normanno nel Liber ad honorem Augusti di Pietro da Eboli (composto tra 1194 e 1197; Berna, Burgerbibliothek ms. 120, II, c. 97r), e ancora hakìm è definito dalle fonti arabe lo imperialis philosophus Teodoro d'Antiochia (m. 1250 ca.) ${ }^{41}$.

\section{IL CONTESTO}

Il Vaticano ebr. 358 è uno straordinario testimone della circolazione del sapere medico arabo-islamico tra gli esponenti della comunità ebraica trapanese. Questa circolazione si inserisce nel quadro della più vasta eredità arabofona degli ebrei dell'isola, ma si giustifica anche in virtù della vocazione geopolitica di Trapani, aperta alla vicina costa africana e al mondo arabo-islamico in generale.

La Trapani della fine del XIII secolo è la città degli Abbate e, come scrive Laura Sciascia, «per quasi due secoli, dalla prima metà del Duecento alla fine del Trecento, il nome e le vicende della famiglia saranno legati strettamente al nome e alle vicende di Trapani: e anche se gli Abbate godranno tutti della cittadinanza di Palermo e avranno interessi e feudi in altre zone dell'isola, il loro nome sarà sempre seguito dall'aggiunta de Trapano, a sottolineare ben più che una provenienza, una simbiosi profonda con la città e le sue strutture che, nel volgere degli anni, arriva quasi ad assumere le caratteristiche di un dominio signorile. In particolare gli Abbate sono legati, come vedremo, a un aspetto determinato della vita della città, al ruolo di punta estrema verso l'Africa, il Maghreb e il mondo islamico che Trapani ebbe per tutta l'età normanna e sveva e che si affievolisce poi dopo il Vespro» ${ }^{42}$.

\footnotetext{
${ }^{40}$ Biberstein KaZimirski, Dictionnaire arabe-français, I, p. 472.

${ }^{41}$ Bar Hebraeus, Ta'rīh muhtașar al-duwal, ed. A. Șāihạānī (Beirut 1898, rist. Beirut 1983), p. 477; su Teodoro d'Antiochia e le tradizioni arabo-islamiche sullo hakìm cfr. Ch. BuRnetT, «Master Theodore, Frederick's II Philosopher», in Federico II e le nuove culture. Atti del XXXI Convegno storico internazionale (Todi, 19-21 ottobre 1994) (Spoleto 1995), pp. 225-85: 248-54.

${ }^{42}$ L. SCIASCIA, «I cammelli e le rose. Gli Abbate di Trapani da Federico II a Martino il Vecchio», in Mediterraneo medievale. Scritti in onore di Francesco Giunta, I-III, Soveria Mannelli 1989, III, pp. 1173-1230: 1174. La studiosa aggiunge anche che: «Il Vespro, infatti, aveva provocato una brusca virata, spostando l'asse degli interessi della città dai rapporti con l'Africa e il mondo musulmano a quelli con la penisola iberica, e facendo del suo porto il naturale punto di approdo delle navi provenienti dalla Catalogna [...]. Il distacco del regno siciliano dall'Aragona rappresenta dunque un momento doppiamente critico, una pausa d'incertezza per la città, che segue quasi immediatamente alla conversione d'interessi verificatasi col Vespro: e tanto più critico per gli Abbate, che sembrano trarre gran parte delle loro ricchezze dai rapporti col mondo musulmano», SCIASCIA, «I cammelli e le rose», p. 1194.
} 
Quanto alla presenza ebraica a Trapani, occorre precisare che durante il XII secolo la città è menzionata sia nelle lettere della Ghenizà del Cairo sia nell'itinerario di Beniamino da Tudela (che riporta un chiaro arabismo nell'indicare il nome locale del corallo: al-murğăn $)^{43}$. Durante l'età normanna e sveva gli statuti della città attestano una promiscuità giuridica, e di conseguenza anche una contiguità di vita civile e religiosa, tra ebrei e cristiani; difatti il legislatore vieta esplicitamente agli esponenti di uno dei due gruppi di testimoniare contro gli altri («Iudaei adversus Christianos nec Christiani adversus Iudaeos in testimonium admittuntur») ${ }^{4}$. Per la seconda metà del XIII secolo qualche lume proviene dalla perduta cancelleria angioina $^{45}$ e dal registro del notaio ericino Giovanni Maiorana, che tuttavia ha il suo focus sul vicino centro di Monte San Giuliano/Erice ${ }^{46}$, ma soprattutto dalla cancelleria aragonese. Difatti sappiamo che qualche mese dopo la copia del nostro codice vaticano, Giacomo d'Aragona nomina sichus e magister legis della universitas trapanese un altro medico ebreo, tale magister Aquacus (29 settembre 1293) ${ }^{47}$.

In definitiva, per i secoli XII e XIII le notizie a disposizione sono estremamente scarse e lacunose, e s'infittiscono progressivamente riguardo ai secoli XIV e $\mathrm{XV}$, grazie alla ricca documentazione archivistica ${ }^{48}$.

Se la Trapani del Due e Trecento è la città degli Abbate, senza dubbio quella del Quattrocento è la città dei Sala (< ar. Șalāḥ), un'importante dinastia di ebrei trapanesi attivi nei commerci mediterranei, che all'occorrenza rivestono il ruolo di ambasciatori nella vicina sponda africana al servizio dei Martini ${ }^{49}$.

${ }^{43}$ The Itinerary of Benjamin of Tudela, p. 71 (testo ebraico), p. 79 (traduzione inglese); su i corallai ebrei trapanesi del XV sec. si vedano Fonti per la storia del corallo nel Medioevo mediterraneo, a cura di A. Sparti, Palermo 1986; A. Precopi Lombardo, L. Novara, «Trapani e i corallai ebrei», Sicilia Archeologica 104, a. 39 (2006), pp. 85-106.

${ }^{44}$ Simonsohn, The Jews in Sicily, I, doc. 198, p. 434.

${ }^{45} \mathrm{Nel} 1272$ un Sulaymān Sacerdoti/Kohen riceve la conferma di un incarico comunitario dal vescovo di Mazara, cfr. Simonsohn, The Jews in Sicily, I, doc. 230, p. 466.

${ }^{46}$ Simonsohn, The Jews in Sicily, I, doc. 279, pp. 510-511; doc. 280, p. 512; doc. 300, pp. 532-533; doc. 322, p. 556.

${ }^{47}$ Simonsohn, The Jews in Sicily, I, doc. 274, p. 505

${ }^{48}$ C. Trasselli, «Sulla diffusione e sull'importanza della cultura e della lingua ebraica in Sicilia, particolarmente in Trapani e in Palermo nel sec. XV», Bollettino del centro di studi filologici e linguistici siciliani 15 (1986), pp. 377-382; E. Ashtor, «The Jews of Trapani in the Later Middle Ages», Studi Medievali 25 (1984), pp. 1-30; A. Scandaliato, «Momenti di vita ebraica a Trapani nel Quattrocento», in A. SCANDALIATO, Judaica minora sicula. Indagini sugli ebrei di Sicilia nel Medioevo (Firenze 2006), pp. 269-333.

${ }^{49}$ S. Fodale, «Un ebreo trapanese ambasciatore dei Martini a Tunisi: Samuele Sala», in Studia Historica et Philologica in honorem M. Batllori (Roma 1984), pp. 255-280; Scandaliato, 
Quanto alla vita culturale della Trapani quattrocentesca Eliyahu Ashtor deprecava, a torto, il basso livello di cultura ebraica della città e aggiungeva che «these people [scil. rich Jews] could support Jewish scholars and render it possible for them to pursue their activities of studying, teaching and writing books» ${ }^{50}$. Lo studioso constatava inoltre che nonostante gli atti dei notai menzionino libri appartenuti ad ebrei trapanesi, vano risultava lo spoglio dei cataloghi di manoscritti alla ricerca di codici composti o copiati a Trapani ${ }^{51}$.

Tuttavia questo giudizio, alquanto affrettato, può essere rivisitato grazie al presente codice, ma anche in virtù delle indagini archivistiche condotte da Angela Scandaliato; ad esempio sappiamo di un copista, Machalufo Chilfa figlio di Xalomo, attivo a Trapani nella seconda metà del XV secolo ${ }^{52}$.

Per quel che concerne l'arte medica anche a Trapani affiora la presenza di qualche medico che ha lasciato traccia delle sue attività nei documenti d'archivio $^{53}$. Come recentemente notato da Shlomo Simonsohn, nella Sicilia ebraica è presente un insolito numero di medici, un'arte che doveva essere ben radicata attraverso pubbliche licenze e una trasmissione all'interno della sfera familiare ${ }^{54}$.

Questo milieu medico plurilingue, ancora tutto da indagare nella sua complessità, trova un riscontro preciso anche nell'evidenza codicologica e mi riferisco, ad esempio, al Vaticano ebr. 361 (ff. 32-166), un codice che contiene trattati medici arabi, prontuari e lessici bilingui, copiato a Palermo nel 1342 da David figlio di 'Eliyya, entrambi definiti ha-Rofe'/'il Medico', ${ }^{55}$, con ogni buona probabilità sia perché esercitanti tale professione sia perché appartenenti alla famiglia de Medico/lu Medicu, una casata fortemente endogama i cui membri vengono costantemente definiti per l'appunto domus Medici o de cognomine de

«Momenti di vita ebraica», pp. 284-297; G. Costantino, «Le relazioni degli ebrei trapanesi con il regno hafside di Tunisi sotto Alfonso V», Mediterranea. Ricerche storiche 5 (dicembre 2008), pp. 505-526.

${ }^{50}$ Ashtor, «The Jews of Trapani», pp. 29-30.

${ }^{51}$ «Some notarial acts dealing with the bequest of Trapanese Jews mention Hebrew books such as the works of Isaac Alfasi and the commentaries of Abraham Ibn Ezra. But one may search in vain in the catalogues of Hebrew MSS. collections for the title of a treatise written or copied in Trapani», Ashtor, «The Jews of Trapani», p. 30.

${ }^{52}$ SCAndaliato, «Momenti di vita ebraica», pp. 333-335.

${ }^{53}$ SCandaliato, «Momenti di vita ebraica», pp. 277-278.

${ }^{54}$ BRESC, Arabi per lingua ebrei per religione, pp. 56-62; Simonsohn, The Jews in Sicily, XVIII, pp. 12159-12166 («Medicine was the first and foremost occupation of Sicilian Jewish professionals»).

${ }^{55}$ Proverbio, «Manoscritti scientifici giudeo-arabi», pp. 354-361. 
Medico, o ancora cognomen et cognacio de Medico, con chiaro e a volte esplicito riferimento al capostipite: il medico Busach/Yișhaq.

A questa importante dinastia di medici palermitani appartiene anche Ahițuv ben Yișhaq un personaggio di primo piano nella Sicilia ebraica di fine Duecento; suo padre era stato medico dell'imperatore Federico II Hohenstaufen, e suo fratello David occupa una posizione simile durante i decenni successivi al Vespro (30 marzo 1282) ${ }^{56}$. Ancora Gaudio, figlio di David, è convocato da Federico III a Messina (11 luglio 1328) ${ }^{57}$, città in cui il sovrano e il figlio giacevano gravemente malati di terzana, e mi sembra probabile che l'appello sottenda la prestazione di cure mediche ${ }^{58}$. Fino alla all'espulsione del 1492 questi «Medici» palermitani svolgeranno le loro attività all'ombra del potere, e grazie al loro status di «medici» potranno rivendicare antichi privilegi, rinnovati ma anche contestati dalle autorità ${ }^{59}$.

In particolare Ahițuv spicca per le sue capacità di letterato e filosofo, attivo nell'isola ancor prima dell'arrivo del mistico e messia Avraham Abulafia (a. 1280). Il suo Poemetto del canestro (Mahberet ha-țene') lo inserisce in un orizzonte culturale che è tutto salvo che provinciale; difatti Ahițuv racconta cinquanta anni prima di Dante un viaggio nell'Oltretomba, che è pretesto per l'esposizione degli articoli della fede ebraica (yesodot 'fondamenti' o 'iqqarim 'principi' $)^{60}$. Ahițuv si fa interprete del pensiero di Maimonide e l'opera intesse legami complessi con la lirica sefardita, ma anche la letteratura ebraica della Roma del primo Trecento.

A questo aspetto della sua produzione si aggiunge anche un'attività di traduttore dall'arabo in caratteri ebraici all'ebraico, che si esplicita nella versione del Trattato sull'arte della logica (Maqāla fì șinā'at al-manțiq) attribuito a Maimonide ${ }^{61}$.

${ }^{56}$ G. Mandalà, «Ahitụu ben Yiṣhaq da Palermo, medico, filosofo e traduttore del secolo XIII», Materia Giudaica 13 (2008), pp. 35-61: 42-49.

${ }^{57}$ Simonsohn, The Jews in Sicily, II, doc. 365, p. 647; doc. 366, pp. 647-48; S. V. Bozzo, Note storiche siciliane del secolo XIV (Palermo 1882), pp. 564-65 e n. 3.

${ }^{58}$ Bozzo, Note storiche siciliane, p. 589; Bresc, Arabi per lingua Ebrei per religione, p. 59.

${ }^{59}$ Mandalà, «Ahịtuv ben Yiṣ̣aq da Palermo», pp. 42-53.

${ }^{60}$ Ho in preparazione un'edizione tradotta e commentata del poemetto; frattanto si vedano $\mathrm{J}$. Schirmann, «Zur Geschichte der hebräischen Poesie in Apulien und Sizilien», Mitteilungen des Forschungsinstituts für hebräische Dichtung 1 (1933), pp. 95-147: 132-147; MANDALÀ, «Ahituv ben Yișhaq da Palermo», pp. 56-59.

${ }^{61}$ L'edizione di riferimento è quella approntata da I. Efros, «Maimonides' Arabic Treatise on Logic Introduction», Proceedings of the American Academy for Jewish Research 34 (1966), pp. 155-160; pp. 9-42 (testo arabo in caratteri ebraici); additamenta di L. V. BERMAN, «Some Remarks on the Arabic Texts of Maimonides' 'Treatise on the Art of Logic'», Journal of the American 
Occorre precisare che questa attività di traduttore non deve essere analizzata esclusivamente dal punto di vista della storia delle idee. Essa è condizionata da un milieu sociolinguistico le cui potenzialità trovano riscontro in campi differenti: alcuni membri della famiglia di Ahițuv, fra cui il fratello David e i nipoti Šemu'el e Gaudio, traducono negli stessi anni dall'arabo in latino antichi privilegi della cancelleria normanna per la élite politico-istituzionale cristiana ${ }^{62}$. A questa cerchia si aggiunge anche un magister Muse medicus iudeus che potrebbe essere identificato con un altro traduttore dall'arabo al latino: Mosè da Palermo che traduce trattati di ippiatria e che è attivo tra Salerno e Napoli, al servizio degli Angiò, durante gli anni ' 70 del sec. XIII ${ }^{63}$.

Questa duplice attività di traduzione, scientifica e amministrativa, è altamente significativa. Nella Palermo di fine Duecento, lontana erede dei fasti normanni, Ahituv e i suoi sono i soli interpreti capaci di affrontare una traduzione dall'arabo, sia esso l'arabo in caratteri ebraici utilizzato da Maimonide o l'arabo dei documenti della cancelleria normanna. Questi due tipi di traduzione si ricollegano al ruolo di interfaccia culturale esercitato da questa importante famiglia ebraica, orientata all'occorrenza sia verso la élite colta delle comunità ebraiche sia verso le esigenze giuridiche delle istituzioni politico-religiose cristiane. Que-

Oriental Society 88 (1968), pp. 340-342. Per la traduzione di Ahițv cfr. I. EFros, «Millot hahiggayôn. Maimonides' Treatise on Logic (Maqālah fì șinā'at al-manțiq). The Original Arabic and Three Hebrew Translations», Proceedings of the American Academy for Jewish Research 8 (1937-38), pp. 1-65: 8-9 (introduzione); 1-136: 67-100 (testo ebraico).

${ }^{62}$ Per le traduzioni dall'arabo in latino cfr. Palermo, Archivio di Stato [= ASP], Diplomatico, Tabulario della Commenda della Magione, n. 224 (Palermo 12 febbraio 1291), Ptholomeus de Capua giudice, Benedictus publicus tabellio grecus et latinus civitatis Panormi notaio. Per un'edizione cfr. C. BRÜHL, Diplomi e cancelleria di Ruggero II (Palermo 1983), pp. 20-21; Simonsohn, The Jews in Sicily, I, doc. 267, p. 497-498; e anche J. Johns, Arabic Administration in Norman Sicily. The Royal Dīwān (Cambridge 2002), p. 101, 121-23, 131-32; regesti: n. 14, p. 304; n. 23, p. 307. ASP, Diplomatico, Tabulario della mensa vescovile di Cefalù, n. 60 (Palermo 5 agosto 1286), Thomasius Grillus giudice, Benedictus publicus tabellio Panormi notaio; cfr. Simonsohn, The Jews in Sicily, I, doc. 257, pp. 490-91; Johns, Arabic Administration, pp. 170171, 184, 203 n. 49 e 51, 209, 279 n. 122; regesto n. 41, p. 313; MANDALÀ, «Ahịtuv ben Yiṣhaq da Palermo», pp. 46-48.

${ }^{63}$ Su Mosè da Palermo si vedano P. Delprato, Trattati di mascalcia attribuiti ad Ippocrate tradotti dall'arabo in latino da Maestro Moisè da Palermo (Bologna 1865); M. Amari, Storia dei Musulmani di Sicilia, $2^{\mathrm{a}}$ ed. a cura di C. A. Nallino, I-III (Catania 1933-39), III, pp. 716, 892 n. 2; D. Trolli, «Le traduzioni di Mosè da Palermo», in Ead., Studi antichi di veterinaria (Parma 1990), pp. 43-57; S. ArIETI, «Mosè da Palermo e le traduzioni dei trattati di mascalcia di Ippocrate Indiano», in Gli ebrei in Sicilia dal Tardoantico al Medioevo. Studi in onore di Monsignor Benedetto Rocco, ed. N. BUCARIA (Palermo 1998), pp. 55-63; per l'identificazione cfr. Mandalà, «Ahịtuv ben Yiṣhaq da Palermo», p. 48. 
sti due livelli di fruizione, apparentemente distinti e separati, sembrano fondersi e confondersi nel caso del Vaticano ebr. 357, un eccezionale testimone della circolazione del Corano traslitterato in caratteri ebraici (XV sec.), all'interno e all'esterno delle comunità ebraiche siciliane ${ }^{64}$.

Spero che questi brevi cenni servano a delineare il contesto in cui è prodotto e fruito il Vaticano ebr. 358 il primo, ma sicuramente non l'unico, manoscritto ebraico trapanese.

Recibido: $02 / 11 / 2010$

Aceptado: 10/05/2011

${ }^{64}$ A. M. Piemontese, «Il corano latino di Ficino e i corani arabi di Pico e Monchates», Rinascimento 36 (1996), pp. 227-273; B. GRÉVIN, «Un témoin majeur du rôle des communautés juives de Sicile dans la préservation et la diffusion en Italie d'un savoir sur l'arabe et l'Islam au $\mathrm{XV}^{\mathrm{e}}$ siècle : les notes interlinéaires et marginales du 'Coran de Mithridate' (ms. Vat. Ebr. 357)», in Chrétiens, juifs et musulmans dans la Méditerranée médiévale. Études en hommage à Henri Bresc, eds. A. Nef, B. Grévin, E. Tixier (Paris 2008), pp. 45-56; Grévin, Mandalà, «Le rôle des communautés juives siciliennes». 